\title{
AGN in ULIRGs and IRQSOs
}

\author{
David J. E. Floyd ${ }^{1}$ \\ ${ }^{1}$ Space Telescope Science Institute, 3700 San Martin Drive, Baltimore, MD 21210, USA \\ email: floyd@stsci.edu
}

\begin{abstract}
I present preliminary results and discussion from a $K$-band imaging study of the host galaxies of samples of ULIRGs and IR-bright QSOs, designed to match in terms of their FIR luminosities and redshift distributions. Large $K$-band bulges are found to be present in all of the quasars, with the majority of the ULIRGs being best-fit by a disc. Despite the apparent difference, a significant overlap exists between the two populations in terms of their nuclear luminosity. A significant unresolved nuclear component is present in all of the objects, and this is found to correlate with the luminosity of the $K$-band host across the sample.
\end{abstract}

\section{Overview}

Using $K$-band UKIRT/UFTI imaging to detect the presence of dynamically relaxed bulges allows a direct test of the viability of an evolutionary link between ULIRGs and QSOs (as proposed by Sanders et al. 1988). I have used a 2D modelling technique (e.g. Floyd et al. 2004; Dunlop et al. 2003; McLure et al. 1999) to accurately recover basic host galaxy parameters, and disentangle host and nuclear flux.

Although two major samples of ULIRG's have recently been imaged with NICMOS (Scoville et al. 2000; Bushouse et al. 2002), the $19.2 \times 19.2^{\prime \prime}$ field-of-view of the NIC2 camera means this data is not suitable for detecting the existence of large-scale bulges. At $z=0.1$ for example, this corresponds to a diameter of $\approx 45 \mathrm{kpc}$. By $z=0.2$ NICMOS fits in a diameter of $80 \mathrm{kpc}$, but this is still insufficient to view the whole of a large galaxy, including background.

UFTI, with its $92^{\prime \prime}$ field-of-view, can easily fit in an entire giant elliptical even at $z=0.1$, and thus allow complete and accurate modelling right down to the low surface brightness wings. Together with the high-resolution $V$-band imaging of HST by Farrah et al. (2001), we have a powerful combination in constraining the role of AGN and starbursts within the ULIRG population.

\section{Discussion}

I have succeeded in uncovering $K$-band host galaxies for all 9 objects for which we obtained suitable imaging. Of these, three of the ULIRG hosts are found to be Freeman disc-like systems, with just 2 hosted by a de Vaucouleurs elliptical. All of the quasar hosts are massive, $L^{\star}$ ellipticals, in keeping with the findings of previous studies of quasar host galaxies. Only one ULIRG has a host this luminous; IRAS02054+0835 with $M_{K}=-25$. It is significant that this is the most $60 \mu \mathrm{m}$-luminous object in our sample. In other words, the one most in need of an explanation for its enormous FIR emission.

\subsection{Kormendy relation}

We find that the sample is consistent with a Kormendy relation with a slope of 3.0. The best-fitting relation of slope 3 (Fig. 1a) has the form:

$$
\mu_{1 / 2}=16.3+3.0 \log _{10} R_{1 / 2}
$$




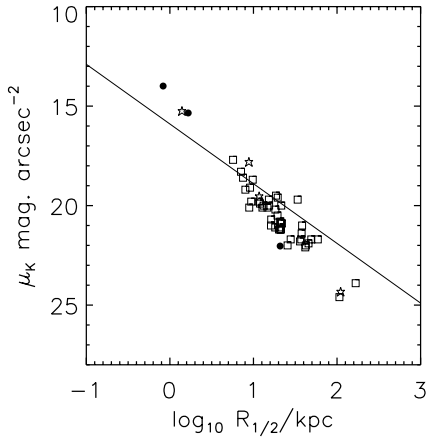

(a)

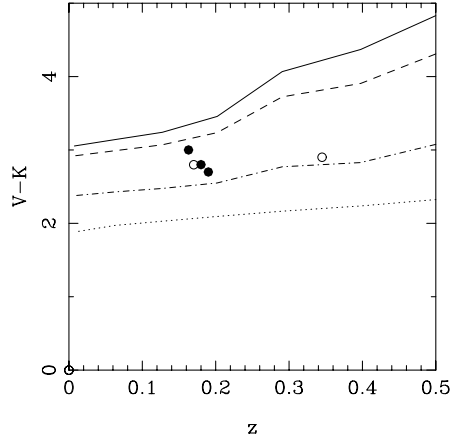

(b)

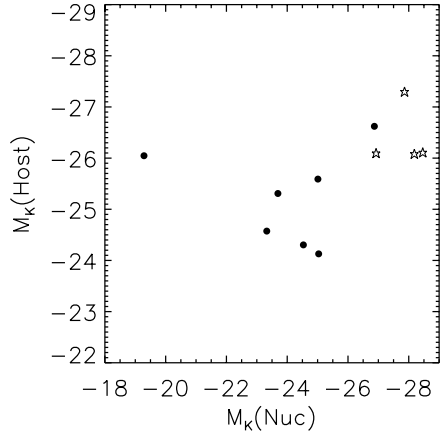

(c)

Figure 1. (a)Best-fit Kormendy relation with a slope of three, showing the ULIRGs (circles), IRQSOs (stars), and the sample of Taylor et al. (1996) (squares). (b) $V-K$ colours predicted by the models of Dunlop et al. (2003) for passive evolution (solid line), with 1\% (dashes), 10\% (dot-dash), and 100\% (dots) lines also given. (c) ULIRG/IRQSO nuclear vs. host magnitudes. The nuclear and host luminosities of the full sample are positively correlated $(\rho=0.67)$ at the $\mathrm{p}=0.05$ level.

\subsection{Host colours}

In Fig. 1b I present basic host colour results for the sample ( $V$-band data from Farrah et al. 2001). The colours of the individual objects are shown along with the passively evolving models of Jimenez et al. (2003), with a variable degree of ongoing star-formation. Full 2D colour maps Floyd (2004) show where star-formation is actively ongoing, and will help us better pin down the colour and age of the underlying stellar population.

\subsection{Nuclear Luminosity}

We find a clear correlation between host and nuclear luminosity across the sample (Fig. 1c), suggesting that there is a connection between the host and the nuclear activity. However, there is no correlation of the nuclear luminosity with the $60 \mu \mathrm{m}$ luminosity, implying that AGN activity cannot be the whole story.

\section{Conclusions}

It is clear that there is some overlap in the two populations, and AGN activity on some level is ubiquitous. In general, however, the ULIRG's do not appear to be on their way to becoming fully-fledged quasars, nor the first-ranked giant ellipticals that we have come to expect to find quasars situated in. It seems likely that such systems are the dusty, still-forming equivalents of the Seyfert galaxies, and while some may end up as quite large $L^{\star}$ ellipticals, many have more in common with the disk galaxy population.

\section{References}

Bushouse, H. A., et al. 2002, ApJS, 138, 1

Dunlop, J. S., et al. 2003, MNRAS, 340, 1095

Farrah, D., et al. 2001, MNRAS, 326, 1333

Floyd, D. J. E. 2004 Ph.D. Thesis, University of Edinburgh

Floyd, D. J. E., et al. 2004, MNRAS, in press

McLure, R. J., et al. 1999, MNRAS, 308, 377

Sanders, D. B., et al. 1988, ApJ, 325, 74

Scoville, N. Z., et al. 2000, AJ, 119, 991 